\title{
A Patient with Diffuse Cutaneous Systemic Sclerosis Complicated by Antineutrophil-cytoplasmic Antibody-associated Vasculitis Exhibiting Honeycomb Lung without Volume Loss
}

\author{
Hiroyuki Yamashita, Yuko Takahashi, Hiroshi Kaneko, Toshikazu Kano and Akio Mimori
}

\begin{abstract}
We herein report the case of a 72-year-old woman with diffuse cutaneous systemic sclerosis ( $\mathrm{SSc}$ ) complicated by antineutrophil cytoplasmic antibody (ANCA)-associated vasculitis who exhibited honeycomb lung without volume loss. On admission, chest computed tomography (CT) revealed honeycomb lung without volume loss in addition to increased density of the partition walls. A renal biopsy revealed global sclerosis and cellular crescent formation. Mononeuritis multiple subsequently occurred, and steroid pulse therapy with cyclophosphamide was administered. Repeat chest CT showed that the honeycomb lung was unchanged; however, overall reduced density of the partition walls was observed. It is necessary to recognise that vasculitis may develop in SSc patients who exhibit honeycomb lung without volume loss.
\end{abstract}

Key words: sclerosis, vasculitis, antineutrophil cytoplasmic antibodies, interstitial lung disease

(Intern Med 53: 801-804, 2014)

(DOI: 10.2169/internalmedicine.53.1599)

\section{Introduction}

Systemic sclerosis (SSc) is a chronic systemic fibrosing disease associated with autoimmune abnormalities. The primary manifestations include three features: tissue fibrosis, autoimmune disorders and microvascular injury. Interstitial lung disease (ILD), principally nonspecific interstitial pneumonitis and less frequently usual interstitial pneumonitis, is observed in up to $75 \%$ of SSc patients (1). Antineutrophil cytoplasmic antibody (ANCA)-associated vasculitis (AAV) is characterized by the presence of systemic necrotising vasculitis of unknown aetiology, which is not a typical finding of SSc. Nevertheless, ANCA is observed in up to $11.7 \%$ of patients with SSc, and several reports have described SSc patients with AAV (2-5). We herein report the case of a 72year-old woman with diffuse cutaneous SSc (dcSSc) complicated by AAV who exhibited honeycomb lung without volume loss.

\section{Case Report}

At 52 years of age, the patient developed Raynaud symptoms and was diagnosed with dcSSc, presenting with sclerosis and pulmonary fibrosis (Fig. 1A). At that time, both upper limbs, including the areas proximal to the elbow joints, showed extensive dermal sclerosis, and the patient was positive for anti-Scl-70 antibodies. In May 2006, she developed a productive cough and exertional dyspnoea. She had no history of smoking. On June 22, she was admitted to our department. On admission, a blood examination revealed the following results: blood urea nitrogen, $24.7 \mathrm{mg} / \mathrm{dL}$; creatinine, $1.0 \mathrm{mg} / \mathrm{dL}$; C-reactive protein, $8.65 \mathrm{mg} / \mathrm{dL}$; perinuclear (P)-ANCA, 44.8 U/mL; and KL-6, $492 \mathrm{U} / \mathrm{mL}$. The results of a urinalysis were as follows: urinary proteins, $2^{+}$; urinary occult blood, $3^{+}$; urinary red blood cells, 30-49/HPF; and erythrocyte casts. The patient's blood pressure was 138/ $70 \mathrm{mmHg}$, and the fundus findings were normal. She displayed no signs of a renal crisis associated with malignant hypertension and had neither haemolytic anemia nor throm- 

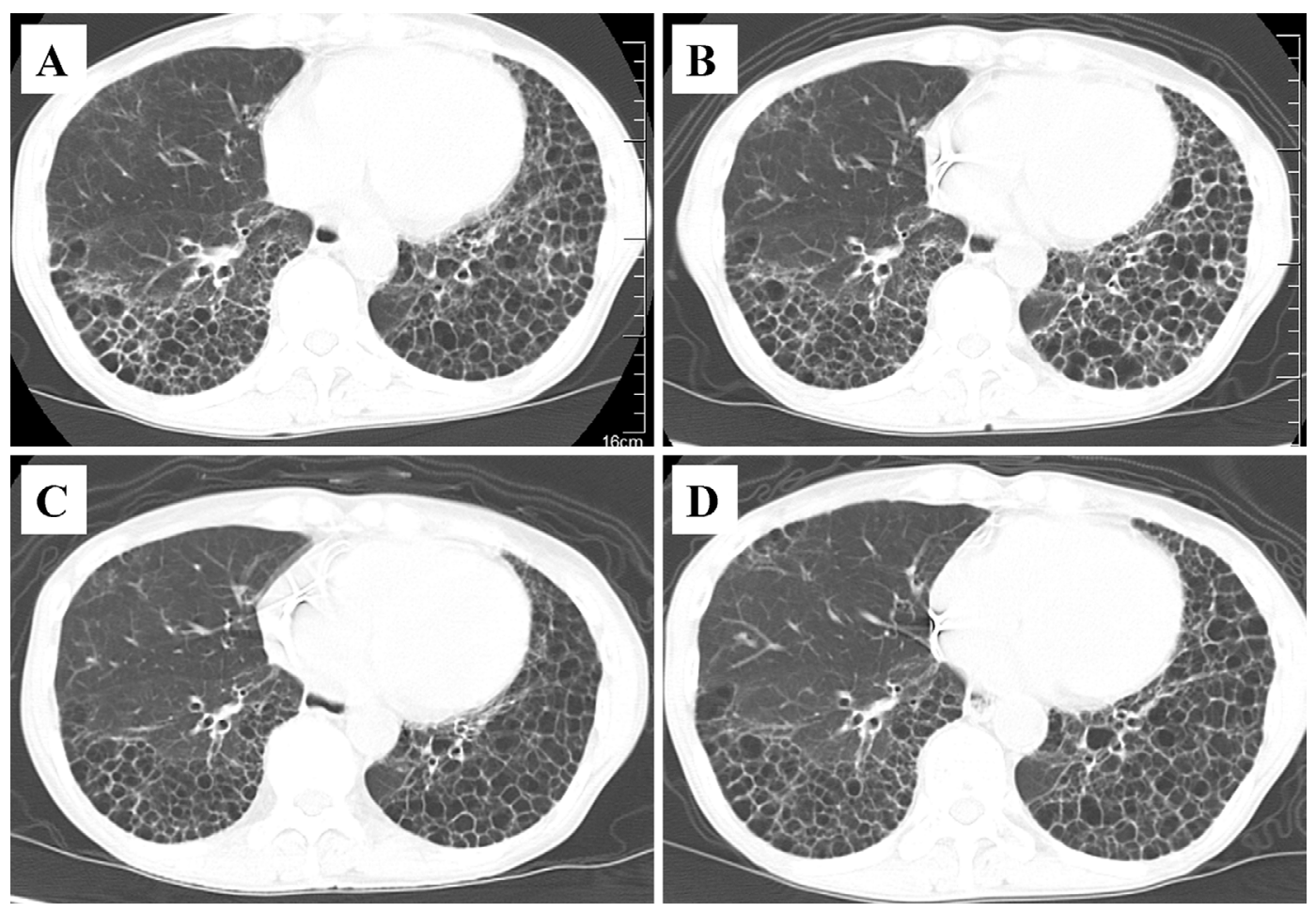

Figure 1. Comparison of the chest CT findings obtained in 2004 (A), on admission (before AAV treatment)(B) in June 2006, after AAV treatment (C) and at the end of 2007 (at AAV relapse) (D). (A) While significant honeycomb lung is observed in both lower lobes, there is little increase in the density of the partition walls. (B) While the honeycomb lung itself shows minimal progression, an increase in the density of the partition walls is apparent. (C) Following the administration of immunosuppressive therapy, partition wall thinning was observed, together with a reduction in density. (D) Few marked changes are noted compared with that observed on the chest CT image obtained after AAV treatment (C).

bocytopenia. Her haptoglobin level was normal at $168.2 \mathrm{mg} /$ $\mathrm{dL}$, and she was negative for concurrent thrombotic microangiopathy. A physical examination showed that the dermal sclerosis had progressed to the trunk. The lung and kidney lesions and reflux esophagitis were the only manifestations of organ involvement. There was no evidence of pulmonary hypertension. A sputum culture revealed Pseudomonas aeruginosa. A respiratory examination demonstrated a normal ventilatory function, although the diffusion capacity was decreased, with a DLCO/VA of $1.67 \mathrm{~mL} / \mathrm{min} /$ $\mathrm{mmHg} / \mathrm{L}(38.5 \%$ of predicted). Chest CT showed honeycomb lung with an increased density of cystic airspace walls without volume loss (Fig. 1B and Fig. 2). A diagnosis of bronchial pneumonia associated with interstitial pneumonia was initially suspected. Therefore, piperacillin and tobramycin were administered. However, the inflammatory responses did not improve. Next, a vasculitic combination was suspected, and a renal biopsy was performed. Global sclerosis and cellular crescent formation were observed, along with the presence of fibrin thrombi. On July 27, left drop foot suddenly occurred. Nerve conduction velocity testing showed the absence of sural nerve conduction attributed to mononeuritis multiplex accompanying the vasculitis. Based on this symptom, the renal biopsy findings and the positive P-ANCA status, a definitive diagnosis of MPA was made.
Steroid pulse therapy was administered, with additional pulse therapy and the intravenous infusion of cyclophosphamide (CPA). The inflammatory responses disappeared, and the urinary sediment test results normalised. Repeat chest CT (Fig. 1C) showed that the honeycomb lung remained unchanged; however, overall thinning and a reduced density of the partition walls were observed. The doses of prednisolone (PSL) and oral CPA were decreased to 10 and $50 \mathrm{mg}$, respectively. Upon discontinuation of CPA at the end of 2007, the patient relapsed. The primary symptom was pyrexia associated with another spike in the inflammatory response (CRP $8.65 \mathrm{mg} / \mathrm{dL}$ ), without concurrent infection. However, the P-ANCA level was normal at less than $1.3 \mathrm{U} /$ $\mathrm{mL}$, and chest CT (Fig. 1D) showed no changes from the findings observed after the previous treatment. No increases were noted in the density of the partition walls in the honeycomb lung. Although treated again, the patient concomitantly acquired refractory atypical mycobacteriosis, pulmonary aspergillosis and cytomegalovirus infection and died in August 2008.

\section{Discussion}

Limited cutaneous SSc is common in cases involving complications of SSc and AAV despite positive results for 

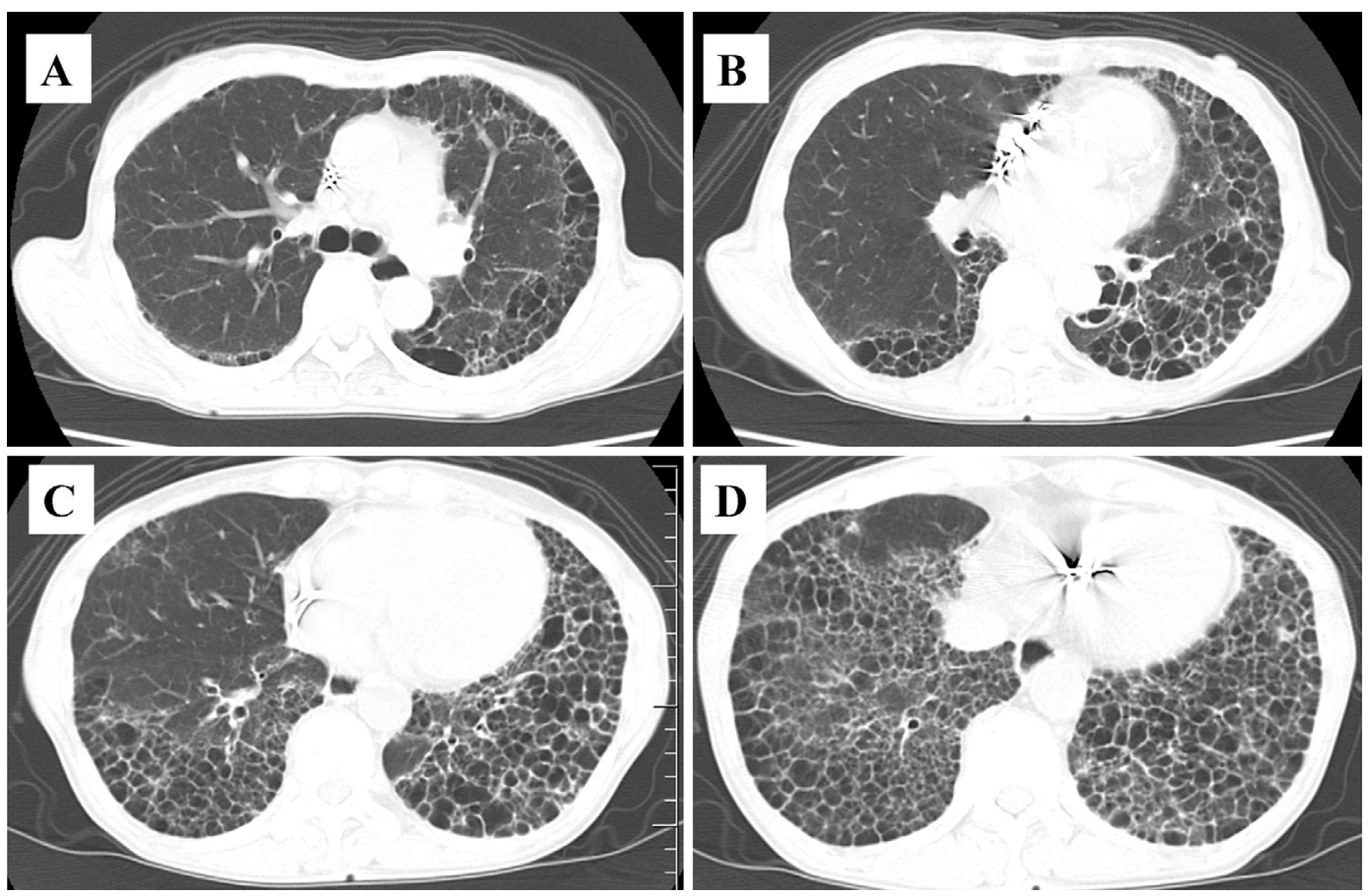

Figure 2. Findings of the chest CT scans obtained before the administration of steroid and CPA therapy on admission. (A-D) Honeycomb lung, with increasing wall density, was observed predominantly in the lower lung fields. The honeycomb lung shows similar findings in the bilateral lower lobes, different from that observed in emphysema, primarily affecting the left upper lobe, with a slight increase in the density of the partition walls. While mild line-form and ground glass-like shadows are observed in the right middle lobe, there are no such findings at other sites.

anti-Scl-70 antibodies (5); however, dcSSc was observed in the present case. Aggregating past cases of SSc complicated by $\mathrm{AAV}$, it is clear, based on immunofluorescence, that $\mathrm{P}$ ANCA accounts for the majority of cases (41/42 cases), as observed in the present patient (5).

Kidney involvement is found in up to $60 \%$ of patients with SSc (6), with scleroderma renal crisis (SRC) occurring in nearly $10 \%$ of cases (7). In SSc patients with rapidly progressive renal failure, it is not easy to distinguish whether the renal failure is the result of SRC or MPA. Although it is important to always carefully make such a distinction, SRC is likely to occur in the onset phase of SSc; therefore, SRC was unlikely to have occurred after that point in the present case.

Rho et al. suggested that anti-Scl-70 antibodies play a role in the development of AAV and represent a significant predictor of AAV in SSc patients (8). Interestingly, pulmonary fibrosis has been described as a manifestation of MPA; sometimes occurring several years before the onset of MPA (9). Such manifestations are attributed not only to recurrent alveolar haemorrhage, but also to an accelerated fibrotic process related to increased oxidative stress mediated by anti-MPO antibodies (10). Therefore, ILD is also a potential manifestation of anti-MPO-associated vasculitis rather than a component of SSc. Mild deterioration of the renal function along with microscopic hematuria was observed from the time the patient was referred to our department and was occasionally accompanied by the emergence of an in- flammatory response. As no abnormal chest shadows were observed on the chest X-rays obtained at another hospital before the patient was referred to our department, the latent vasculitis that had existed for many years may have been allowed to proceed to destroy the structure of the lung in the form of a honeycomb lung.

As the vasculitis activity increased, cellular infiltration may have occurred in the lungs due to the vasculitis in the form of increased wall density in the honeycomb lung.

According to the images, the differentiaal diagnosis in this case includes bacterial pneumonia, pulmonary congestion and pulmonary alveolar haemorrhage. A diagnosis of bacterial pneumonia was negative, based on the lack of response to antimicrobial drugs. The possibility of pulmonary congestion was ruled out, as cardiac ultrasonography showed the cardiac function to be maintained and the administration of steroids was markedly effective. The presence of pulmonary alveolar haemorrhage was also ruled out, as the patient had no relevant symptoms, such as bloodstained sputum or an increase in DLCO/VA. Typically, interstitial pneumonia associated with SSc primarily involves nonspecific interstitial pneumonia, with usual interstitial pneumonia (UIP) being rare $(11,12)$. Moreover, it has been suggested from the aforementioned course that interstitial pneumonia is caused by AAV. On the other hand, pulmonary function studies in patients with UIP typically demonstrate a restrictive pattern, while chest radiographs show diffuse reticular opacity associated with a reduced lung volume. How- 
ever, in this case, when the chest radiograms were compared over time, no signs of a decreased lung volume, such as progressive diaphragmatic elevation, were observed and the patient's vital capacity $(\% \mathrm{VC}=96.7 \%)$ was normal on lung function testing, suggesting no evidence of volume loss. Such findings are rare in patients with honeycomb lung. Accordingly, taking these observations together, we propose that the presence of latent vasculitis be considered when patients with scleroderma exhibit a "honeycomb lung without volume loss."

\section{Conclusion}

It is necessary to recognise that vasculitis may develop in SSc patients who exhibit honeycomb lung without volume loss.

The authors state that they have no Conflict of Interest (COI).

\section{Acknowledgement}

The present work was supported by Grants-in-Aid for Research on Intractable Diseases from the Ministry of Health, Labour and Welfare of Japan.

\section{References}

1. Steen VD, Medsger TA. Changes in causes of death in systemic sclerosis, 1972-2002. Ann Rheum Dis 66: 940-944, 2007.

2. Akimoto S, Ishikawa O, Tamura T, Miyachi Y. Antineutrophil cytoplasmic autoantibodies in patients with systemic sclerosis. Br J
Dermatol 134: 407-410, 1996.

3. Casari S, Haeney M, Farrand S, Herrick A. Antineutrophil cytoplasmic antibodies a "red flag" in patients with systemic sclerosis. J Rheumatol 29: 2666-2667, 2002.

4. Chifflot H, Fautrel B, Sordet C, Chatelus E, Sibilia J. Incidence and prevalence of systemic sclerosis: a systematic literature review. Semin Arthritis Rheum 37: 223-235, 2008.

5. Quéméneur T, Mouthon $L$, Cacoub $P$, et al. Systemic vasculitis during the course of systemic sclerosis: report of 12 cases and review of the literature. Medicine (Baltimore) 92: 1-9, 2013.

6. Cannon PJ, Hassar M, Case DB, Casarella WJ, Sommers SC, LeRoy EC. The relationship of hypertension and renal failure in scleroderma (progressive systemic sclerosis) to structural and functional abnormalities of the renal cortical circulation. Medicine (Baltimore) 53: 1-46, 1974.

7. Teixeira L, Mouthon L, Mahr A, et al. Mortality and risk factors of scleroderma renal crisis: a French retrospective study of 50 patients. Ann Rheum Dis 67: 110-116, 2008.

8. Rho YH, Choi SJ, Lee YH, Ji JD, Song GG. Scleroderma associated with ANCA-associated vasculitis. Rheumatol Int 26: 465-468, 2006.

9. Tzelepis GE, Kokosi M, Tzioufas A, et al. Prevalence and outcome of pulmonary fibrosis in microscopic polyangiitis. Eur Respir J 36: 116-121, 2010.

10. Guilpain P, Chéreau C, Goulvestre C, et al. The oxidation induced by antimyeloperoxidase antibodies triggers fibrosis in microscopic polyangiitis. Eur Respir J 37: 1503-1513, 2011.

11. Fischer A, Swigris JJ, Groshong SD, et al. Clinically significant interstitial lung disease in limited scleroderma: histopathology, clinical features, and survival. Chest 134: 601, 2008.

12. Bouros D, Wells AU, Nicholson AG, et al. Histopathologic subsets of fibrosing alveolitis in patients with systemic sclerosis and their relationship to outcome. Am J Respir Crit Care Med 165: 1581, 2002.

(C) 2014 The Japanese Society of Internal Medicine http://www.naika.or.jp/imonline/index.html 